Usán Supervía, P., Salavera Bordás, C. \& Domper Buil, E. (2018). ¿Cómo se interrelacionan las variables de burnout, engagement y autoeficacia académica? Un estudio con adolescentes escolares. Revista Electrónica Interuniversitaria de Formación del Profesorado, 21(2), 141-153.

\title{
¿Cómo se interrelacionan las variables de burnout, engagement y autoeficacia académica? Un estudio con adolescentes escolares
}

Pablo Usán Supervía, Carlos Salavera Bordás y Elena Domper Buil

Facultad de Educación, Universidad de Zaragoza.

\section{Resumen}

El objetivo del presente trabajo fue analizar la relación entre burnout, engagement y autoeficacia académica en una muestra de 1756 alumnos $(M=14.47$; $D T=1.63)$ pertenecientes a 12 centros de Educación Secundaria Obligatoria (ESO). Los instrumentos utilizados fueron las versiones españolas del Maslach Burnout Inventory - Student Survey (MBI-SS), la Utrecht Work Engagement Scale - Student (UWES-S) y la Escala de Autoeficacia Percibida Específica de Situaciones Académicas (EAPESA). Los resultados mostraron relaciones significativas inversas entre el agotamiento físico/emocional y cinismo del burnout con el vigor y dedicación del engagement de la misma manera que relaciones positivas entre la autoeficacia académica con el vigor, dedicación y absorción, en una línea de conductas más autodeterminadas. Se desprende la importancia de la promoción de comportamientos adaptativos que mejoren el bienestar y vida académica de los estudiantes en sus centros educativos.

\section{Palabras clave}

Burnout; engagement; autoeficacia; estudiantes.

\section{How is the relationship between burnout, engagement and academic self-efficacy? A study in teenage students.}

\section{Contacto:}

Pablo Usán Supervía, pusan@unizar.es, C/Pedro Cerbuna n¹2, 50009. Facultad de Educación. Universidad de Zaragoza. OPICS. Observatorio para la investigación e innovación en Ciencias Sociales. 


\section{Abstract}

The aim of this research was to analyze the relationship between burnout, engagement and academic self-efficacy in a sample of 1756 students $(M=14.47, S D=1.63)$ belonging to 12 secondary education highschools. The instruments used were the Spanish versions of the Maslach Burnout Inventory - Student Survey (MBI-SS), Utrecht Student Student Scale (UWES-S) and the Specific Perceived Self-Efficacy Scale of Academic Situations (EAPESA). The results showed inverse relationship between physical\&emotional effort and cynicism with vigor and dedication in the same way those positive relationships between academic self-efficacy with vigor, dedication and task absorption, in a more adaptive behaviors. The importance of the promotion of adaptive behaviors that improve the well-being and academic life of the students in their educational centers is revealed.

\section{Key words}

Burnout; engagement; self-efficacy; students.

\section{Introducción}

Durante el proceso educativo en los centros escolares se producen numerosas situaciones contextuales y personales que afectan de manera significativa a los estudiantes en su proceso de formación, más si cabe en enseñanzas medias, coincidiendo con la adolescencia, etapa de vital importancia en la vida de una persona y paso previo a la edad adulta (GómezFraguela, Fernández, Romero y Luengo, 2008).

De este modo, ciertos estudiantes durante su escolaridad, pueden no disponer de las estrategias y competencias necesarias para las exigencias de la vida académica y demandas del curso escolar en el que se hallen inmersos pudiendo llegar a experimentar sensaciones y percepciones varias como la asunción de una actitud negativa de desvalorización y pérdida de interés en sus estudios, aparición de dudas acerca de la propia capacidad personal en sacarlos adelante o, incluso, agotamiento físico y psíquico (Palacio, Caballero, González, Gravini, 2012; Contreras, 2012; Bresó, 2008) que pueden desembocar en una desmotivación absoluta y posterior abandono de sus estudios (De Andrade, 2007; Musitu, Giménez y Burgui, 2012). La aparición de dichas manifestaciones se conoce como síndrome de burnout académico (Schaufeli, Martínez, Marques Pinto, Salanova y Bakker, 2002; Schaufeli y Salanova, 2007) caracterizado por tres dimensiones principales: cansancio emocional, cinismo y eficacia. La primera de ellas, se relaciona con el agotamiento físico y desgaste emocional que puede llegar a tener un alumno en un momento dado más o menos persistente durante su etapa escolar; la segunda, el cinismo, hace referencia a la más pura indiferencia o falta de interés hacia los estudios y, por último, la eficacia, se vincula con la aptitud para el desempeño de las tareas escolares.

De esta manera, el burnout académico se ha relacionado con un bajo rendimiento escolar (Salanova, Martínez y Llorens, 2014), altos niveles de estrés (Extremera, Durán, y Rey, 2007), bajos niveles de autoeficacia (Durán, Extremera, Rey, Fernández-Berrocal, y Montalbán, 2006; Rodriguez-García, Sola-Martínez y Fernandez-Cruz, 2017) así como, en definitiva, escasa felicidad académica durante la etapa escolar del alumno (Salanova, Martínez, Bresó, Llorens y Grau, 2005).

Por otra parte, el engagement académico, se considera un estado psicológico proveniente de la psicología positiva fundamentado en el buen funcionamiento y fortalezas del individuo frente a las situaciones de estrés y/o agotamiento (Seligman y Csikszentmihalyi, 
2000; Seligman, Steen, Park y Peterson, 2005). En un sentido contrario al burnout, el engagement académico queda caracterizado por el vigor, la dedicación y la absorción. El primero de ellos, alude a altos niveles de energía y resistencia en la demanda escolar, la inversión de esfuerzos en el trabajo así como la persistencia frente a las dificultades; el segundo, la dedicación, hace referencia a la involucración y compromiso en el trabajo así como por sentimientos de entusiasmo e importancia y, por último, la absorción, resulta un estado de inmersión en la tarea que satisface y colma las expectativas del alumno (Schaufeli y Bakker, 2004).

De esta manera, el engagement académico se asocia con un óptimo rendimiento académico (Vera et al., 2014), altos niveles de percepción de autoeficacia académica (Bresó, Schaufeli y Salanova, 2011) e inteligencia emocional (Extremera et al., 2007), una baja prevalencia de abandono escolar (Salanova et al., 2014) así como, en términos generales, la felicidad académica de los estudiantes (Moyano y Riaño-Hernández, 2013).

Con todo esto, las diversas investigaciones han puesto de manifiesto la existencia de una relación inversa entre las dimensiones que caracterizan al burnout académico y las que responden al engagement académico (Salanova, Del Líbano, Llorens y Schaufeli, 2014).

En este aspecto, se considera que las dimensiones del burnout referidas al agotamiento físico/emocional y el cinismo se contraponen a las dimensiones del engagement, como son el vigor y la dedicación (Salanova, Bresó y Schaufeli, 2005; Salanova, Schaufeli, Martínez y Bresó, 2009). Específicamente, se ha propuesto que las categorías de cansancio emocional y vigor pueden considerarse extremos bipolares de una dimensión que se denominaría energía, al mismo tiempo que las categorías de cinismo y dedicación podrían entenderse como los extremos opuestos de otra dimensión la cual se denominaría identificación (González-Romá, Schaufeli, Bakker, y Lloret, 2006).

Por lo tanto, el engagement académico se consideraría un patrón conductual adaptativo de los alumnos caracterizado por la implicación, satisfacción y desempeño de la propia actividad académica mientras que, el burnout académico, representaría un patrón conductual desadaptativo y opuesto determinado por la fatiga, insatisfacción y desencanto hacia los estudios.

Por otra parte, siguiendo con la teoría social cognitiva, Bandura (1999) define la autoeficacia como la "creencia de que uno es capaz de organizar y ejecutar cursos de acción que requieran de logro y el manejo de situaciones específicas o tareas". En este sentido, se afirma que la autoeficacia influye en las conductas, elección de actividades, patrones de pensamiento así como en las reacciones emocionales determinando el esfuerzo y dedicación que invierten las personas en una actividad concreta, dotando de protagonismo al sujeto y permitiendo que éste sea el productor de su propio futuro y no un predictor del mismo (Brannon, 2001). De esta manera, altos niveles de autoeficacia tienden a prevenir la experiencia del estrés y el agotamiento desembocando en un incremento de las aptitudes personales del alumno en la valoración de aquello que le resulta amenazante y muy estresante en su vida académica (Salanova et al., 2009).

Por otro lado, Bresó et al. (2005) mostraron evidencias empíricas de la relación del burnout y engagement con la baja autoeficacia en estudiantes a través de dos modelos en espiral: el primero, positivo hacia arriba, hace referencia a la relación de mayor éxito académico con mayor autoeficacia y engagement previniendo, de este modo, la experiencia del estrés y baja autoeficacia; el segundo, negativo hacia abajo, efectuado por la relación de una baja autoeficacia con la aparición del síndrome de burnout académico en adolescentes escolares. 
Con todo ello, y siguiendo a Caballero, Abello y Palacio (2007), resultan necesarios más estudios que indaguen en el estudio de las relaciones entre los constructos psicológicos de burnout y engagement en la vida académica de los estudiantes así como el rol mediador de la autoeficacia en aras de la comprensión y análisis de la interrelación entre ellos. De este modo, el objetivo del estudio se centra en analizar la relación entre el burnout, el engagement y la autoeficacia académica en una muestra de adolescentes escolares. En base al objetivo anteriormente enunciado, se postulan dos hipótesis:

(a) Aquellos estudiantes con unas mayores prevalencias de vigor y dedicación en su vida académica presentarán menores niveles de agotamiento físico/emocional y cinismo, reflejado en una relación significativa inversa, siguiendo una línea de conductas más adaptativas.

(b) Aquellos estudiantes que obtengan mayores niveles de autoeficacia académica se relacionarán positivamente con el vigor, dedicación y absorción actuando, negativamente, con sus niveles de agotamiento físico/emocional y cinismo.

\section{Método}

\section{Diseño}

El presente trabajo, de acuerdo con Ato, López y Benavente (2013), consistió en un estudio ex post-facto de carácter prospectivo con un diseño descriptivo simple, respondiendo a un muestreo aleatorio simple.

\section{Muestra}

El estudio estuvo compuesto por 1756 estudiantes de género masculino ( $N=914 ; 52.05 \%$ ) y femenino $(N=842 ; 47.94 \%)$ pertenecientes a 12 centros públicos de Educación Secundaria Obligatoria (ESO) con edades comprendidas entre los 12 y 18 años $(M=14.47 ; D T=1.63)$. En la tabla 1 se exponen las características sociodemográficas de la muestra.

Tabla 1.

Edad y curso académico de los estudiantes.

\begin{tabular}{cccc}
\cline { 3 - 4 } & & $\mathbf{N}$ & $\%$ \\
\hline Edad & 12 años & 307 & 17.48 \\
& 13 años & 293 & 16.68 \\
& 14 años & 403 & 22.94 \\
& 15 años & 417 & 23.74 \\
& 16 años & 269 & 15.31 \\
& 17 años & 56 & 3.18 \\
\hline \multirow{2}{*}{ Curso académico } & 18 años & 11 & 0.62 \\
\hline & $1^{\circ}$ ESO & 338 & 19.24 \\
& $2^{\circ}$ ESO & 436 & 24.82 \\
& $3^{\circ}$ ESO & 567 & 32.28 \\
& $4^{\circ}$ ESO & 415 & 23.63 \\
\hline
\end{tabular}




\section{Instrumentos de evaluación}

Para recoger la información y aproximarnos al objetivo establecido se utilizaron tres cuestionarios.

Con la finalidad de percibir el burnout académico de los estudiantes se utilizó la versión española del Maslach Burnout Inventory - Student Survey (MBI-SS) (Schaufeli et al., 2002). Dicha cuestionario se compone de 15 ítems repartidos en tres dimensiones: agotamiento físico/emocional (5) ( $\alpha=.81)$ (Ej. "Estudiar o ir a clase todo el día es una tensión para mí); cinismo (4) ( $\alpha=.79$ ) (Ej. "He perdido entusiasmo por mis estudios") y eficacia ( 6 ) ( $\alpha=.77$ ) (Ej. "Me estimula conseguir objetivos en mis estudios"). Las respuestas responden al grado de acuerdo o desacuerdo con los ítems en una escala tipo Likert que oscila desde "Muy en desacuerdo" (1) hasta "Muy de acuerdo" (5). La fiabilidad del instrumento original denota un alfa de Cronbach de .80 mientras que en nuestra investigación otorgó una prevalencia de .82 .

Para averiguar el engagement académico de los estudiantes se partió de la versión española del Utrecht Work Engagement Scale - Student (UWES-S) (Schaufeli et al., 2002). El instrumento está compuesto por 17 ítems los cuales se dividen en tres dimensiones: vigor (6) $(\alpha=.74)$ (Ej. "Mis tareas como estudiante me hacen sentir lleno de energía); dedicación (5) $(\alpha=.79)$ (Ej. "Mis estudios son un reto para mí") y absorción (6) $(\alpha=.75)$ (Ej. "Soy feliz cuando realizo mis tareas como estudiante"). Las respuestas oscilan desde "Muy en desacuerdo" (1) hasta "Muy de acuerdo" (5) en una escala tipo Likert. El instrumento original denota un alfa de Cronbach de .80 mientras que en nuestra investigación otorgó una prevalencia de .76 .

Por último, con la finalidad de evaluar la percepción de autoeficacia académica de los participantes en el estudio se utilizó la Escala de Autoeficacia Percibida Específica de Situaciones Académicas (EAPESA) propuesta por García et al., (2010). La escala unifactorial está constituida por 10 ítems (Ej: "Pienso que tengo capacidad para comprender bien y con rapidez una materia") en una escala tipo Likert con puntuaciones desde "Muy en desacuerdo" (1) hasta "Muy de acuerdo" (5), mostrando un Alfa de Cronbach original de .91 mientras que, en nuestra investigación, resultó de .88 reflejando una adecuada fiabilidad.

\section{Procedimiento}

En la realización del estudio se contó con la aprobación de los diferentes centros de educación secundaria así como de los padres/tutores de los alumnos por medio de consentimiento informado para poder participar en la investigación. Un día de la semana, acordado previamente con jefatura de estudios, se llevó a cabo la cumplimentación de los cuestionarios en cada una de las clases del mismo centro. Todos los sujetos y sus padres/tutores fueron previamente informados de la naturaleza del estudio participando de manera voluntaria y respetando, de este modo, las directrices éticas de la Declaración de Helsinki (AMM, 2000) en todos sus términos.

\section{Análisis de datos}

Se procedió a realizar una estadística descriptiva con la finalidad de conocer los datos sociodemográficos de la muestra así como las diferentes variadas estudiadas. Posteriormente, se llevaron a cabo correlaciones entre las variables burnout, engagement y autoeficacia, procesadas y analizadas mediante el programa estadístico IBM SPSS V22.0. Finalmente, se planteó un modelo de ecuaciones estructurales mediante el método de extracción de máxima verosimilitud que permitiese validar y cuantificar las relaciones causales entre el burnout, engagement y autoeficacia académica a través del programa 
AMOS v24. Para todas las operaciones, se tuvo en cuenta un nivel de significación $p \leq 0,05$ trabajándose con un nivel de confianza del $95 \%$.

\section{Resultados}

A continuación se presentan los resultados obtenidos de las diferentes variables estudiadas:

\section{Variables descriptivas}

Tal y como se refleja en la tabla 2, las puntuaciones de las diferentes dimensiones resultaron heterogéneas. La variable de autoeficacia académica $(M=3.52)$ obtuvo mayor puntuación que el resto de dimensiones que componen el burnout y el engagement. En el primero, destaca el agotamiento físico/emocional $(M=3.19)$ por encima del cinismo $(M=2.14)$. Respecto al segundo, destaca la dimensión de dedicación $(M=3.21)$ por delante de la absorción $(M=2.84)$ y el vigor $(M=2.75)$.

Tabla 2.

Descriptivos de las variables de burnout, engagement y autoeficacia académica.

\begin{tabular}{cccc}
\cline { 2 - 4 } & Media & DT & $\begin{array}{c}\text { Alfa de } \\
\text { Cronbach }\end{array}$ \\
\hline Burnout Agotamiento físico/emocional & 3.19 & .96 & .81 \\
Cinismo & 2.14 & 1.06 & .79 \\
Eficacia & 3.30 & .78 & .77 \\
\hline Engagement Vigor & 2.75 & 1.02 & .74 \\
Dedicación & 3.21 & 1.02 & .79 \\
Absorción & 2.84 & .90 & .75 \\
\hline Autoeficacia Autoeficacia académica & 3.52 & .89 & .78 \\
\hline
\end{tabular}

\section{Relación entre las variables de burnout, engagement y autoeficacia}

Analizando las variables de burnout, engagement y autoeficacia académica se apreciaron correlaciones significativas entre varias de ellas (véase Tabla 3).

De esta manera, entre las dimensiones del burnout académico, destacó la significación entre el agotamiento físico/emocional y el cinismo $(r=.328)$. Adentrándonos en el engagement académico, las tres dimensiones se relacionaron significativamente entre sí siendo el vigor y dedicación $(r=.722)$ la correlación más alta hallada. Aunando ambas variables, destaca la relación negativa del agotamiento físico/emocional y el cinismo del burnout con el vigor $(r=-323$ y $r=-.292)$ dedicación $(r=.303$ y $r=.470)$ y absorción $(r=.234$ y $r=.330$ ) respectivamente, de la variable de engagement académico.

En referencia a la variable de autoeficacia académica, se denotaron relaciones significativas negativas de la misma con el agotamiento físico/emocional $(r=-.146)$ y cinismo $(r=-.489)$ del burnout así como, de manera positiva, en las dimensiones de vigor $(r=.601)$, dedicación $(r=.321)$ y absorción ( $r=.426)$ del engagement, respectivamente. 
Tabla 3.

Relación entre variables de burnout, engagement y autoeficacia.

\begin{tabular}{|c|c|c|c|c|c|c|c|}
\hline & \\
\hline & & 1 & 2 & 3 & 4 & 5 & 6 \\
\hline \multicolumn{8}{|c|}{ Burnout $\begin{array}{ll}1 . & \text { Agotamiento } \\
\text { físico/emocional }\end{array}$} \\
\hline & 2. Cinismo & $.328 * *$ & 1 & & & & \\
\hline & 3. Eficacia profesional & $-.158 *$ & $-.489 * *$ & 1 & & & \\
\hline \multicolumn{2}{|c|}{ Engagement 4. Vigor } & $-.323 * *$ & $-.292 * *$ & $.487^{* *}$ & 1 & & \\
\hline \multicolumn{2}{|r|}{ 5. Dedicación } & $-.303 * *$ & $-.470 * *$ & $.623^{* *}$ & $.722 * *$ & 1 & \\
\hline \multicolumn{2}{|r|}{ 6. Absorción } & $-.234^{* *}$ & $-.330 * *$ & $.500 * *$ & $.709 * *$ & $.707^{* *}$ & 1 \\
\hline \multicolumn{2}{|c|}{ Autoeficacia 7. Autoeficacia académica } & $-.146^{*}$ & $-.348 * *$ & $.601 * *$ & $.321 * *$ & $.426 * *$ & $.327^{* *}$ \\
\hline
\end{tabular}

** La correlación es significativa al nivel 0.01 (bilateral)

* La correlación es significante al nivel 0.05 (bilateral)

\section{Modelo de ecuaciones estructurales entre las variables de burnout, engagement y autoeficacia}

Por último, se realizó un modelo de ecuaciones estructurales entre las variables de burnout, engagement y autoeficacia académica de los estudiantes de Secundaria. En la Figura 1, se muestra el resultado del análisis con ecuaciones estructurales mediante el método de extracción de máxima verosimilitud, confirmando la adecuación del modelo compuesto por los constructos planteados en el estudio, el cual, refleja una alta correlación inversa entre autoeficacia académica y burnout ( $r=-.97$ ), lo que nos indicaría que altos niveles de burnout llevan aparejada una baja autoeficacia académica. En esta misma línea, se encontró una correlación positiva ( $r=.34$ ) entre dicha autoeficacia y el engagement académico (véase figura 1). Por lo que se refiere al ajuste del modelo, los diversos índices fueron adecuados, por lo que se puede afirmar que el modelo propuesto acerca de la estructura factorial entre el burnout, el engagement y la autoeficacia académica resulta sustentable: $\chi^{2}(9)=90.950$, $\mathrm{p}<0.001 ; \chi 2 / \mathrm{gl}=10.106$; Índice de ajuste comparativo $=0.97$; Índice de ajuste normado = 0.96; Índice de Tucker-Lewis = 0.92; Error de aproximación medio por grado de libertad = 0.98 , IC $95 \%$ (0.081-0.113).

\section{Discusión y conclusiones}

El objetivo del estudio fue analizar la relación entre el burnout, el engagement y la autoeficacia académica en adolescentes escolares pertenecientes a diferentes centros escolares en etapa de Educación Secundaria Obligatoria.

La primera hipótesis que se postuló fue, precisamente, que aquellos estudiantes con unas mayores prevalencias de vigor y dedicación en su vida académica presentarían menores niveles de agotamiento físico/emocional y cinismo, reflejado en una relación significativa inversa, siguiendo una línea de conductas más adaptativas.

Dicha hipótesis se cumplió; los resultados de nuestra investigación revelaron la relación significativa existente entre unos mayores niveles de vigor y dedicación de los estudiantes, en la variable de engagement, unidos con unos menores niveles de agotamiento físico/emocional y cinismo, por parte del burnout. 


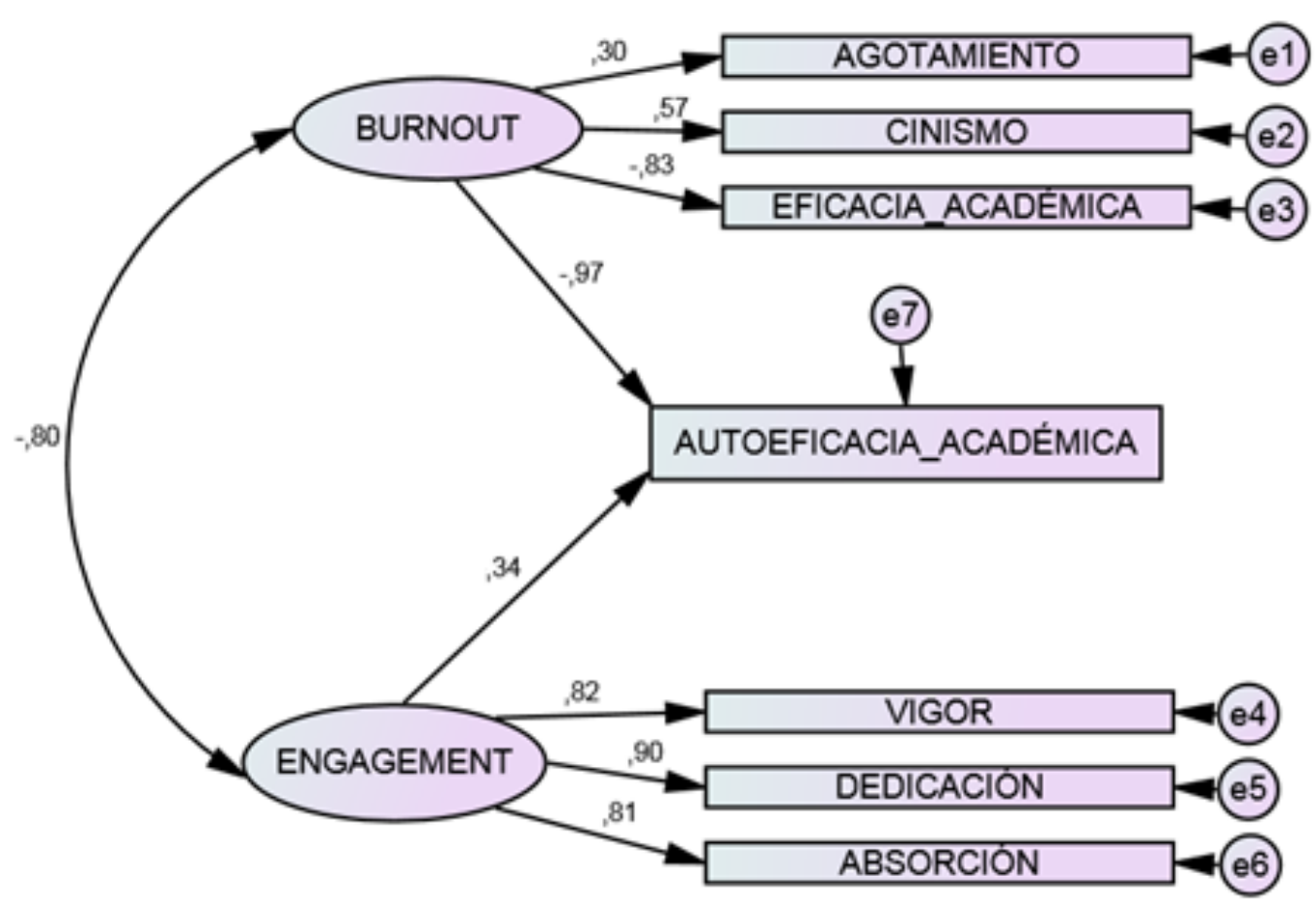

Figura 1. Modelo de ecuaciones estructurales entre burnout, engagement y autoeficacia

De esta manera, aquellos alumnos con altos niveles de energía, resistencia y constancia ante la demanda escolar al mismo tiempo que comprometidos e involucrados con la vida académica, presentaron un menor desgaste físico y emocional así como falta de interés hacia sus estudios.

Diferentes estudios avalan nuestros resultados en una línea de conductas más adaptativas. Salanova, Del Líbano, Llorens y Schaufeli (2014) ponen de manifiesto la relación inversa entre las dimensiones que determinan el burnout y las que caracterizan el engagement, incluida la absorción por los estudios, tal y como también acontece los resultados de nuestra investigación. De este modo, el alumnado se encontraría, además de lo expuesto, en un estado de inmersión en la tarea en la cual se sentiría cómodo, confiado y exitoso solventando las demandas académicas que le van surgiendo. Salanova, Martínez y Llorens (2014) afirman que el óptimo funcionamiento académico se encuentra relacionada negativamente con el burnout y positivamente con el engagement lo cual quiere decir que los estudiantes que se sientan vigorosos, eficaces y absorbidos por el desarrollo de sus tareas académicas y curso escolar en el que se hallen inmersos se desempeñan mejor que quienes experimentan lo contrario (Schaufeli y Bakker, 2004).

Otros estudios, mantienen esta relación inversa entre el burnout y el engagement académico unido a altos niveles de estrés y bajo rendimiento académico (Extremera, Durán y Rey, 2007; Parra, 2010; Salavera y Usán, 2017). De este modo, estos alumnos no sólo mostrarían un mayor agotamiento físico/emocional y cinismo sino, también, altos niveles de desajuste emocional acarreando estrés que podría repercutir en su rendimiento escolar.

Desde una vertiente contraria, otros trabajos aluden a un mayor vigor y dedicación unidos a una mayor inteligencia emocional, un mayor rendimiento académico así como una escasa prevalencia de abandono de los estudios (Bresó, Schaufeli y Salanova, 2011; Extremera et al., 2007) que, en definitiva, repercuten en el bienestar personal y felicidad de los 
estudiantes en aras de la progresión escolar en su vida académica (Moyano y RiañoHernández, 2013; Vera et al., 2014).

La segunda hipótesis de nuestro estudio aludía a la posible relación de la autoeficacia académica de los estudiantes con su vigor, dedicación y absorción en detrimento de su agotamiento físico/emocional y cinismo.

La hipótesis establecida se cumplió; los resultados de nuestra investigación arrojaron una fuerte relación entre la autoeficacia y los niveles de vigor, dedicación y absorción, del engagement académico, en detrimento del agotamiento físico/emocional y cinismo del burnout académico, respectivamente. Además, el modelo de ecuaciones estructurales, definió la relación positiva entre la autoeficacia y el engagement del mismo modo que, de manera inversa, la autoeficacia con el burnout.

De esta manera, aquellos alumnos con mayores niveles de percepción de autoeficacia en sus tareas y cometidos escolares manifiestan mayores niveles de energía, constancia y compromiso que aquellos que muestran mayores prevalencias de agotamiento físico/emocional y cinismo hacia la demanda escolar.

Resultan escasos los estudios que relacionan directamente estas tres variables. Bresó et al., (2008) destaca la inequívoca relación de la autoeficacia con el burnout y el engagement académico. Por un lado, establece una vinculación entre la percepción de autoeficacia académica y el vigor, dedicación y absorción de los estudiantes adolescentes unido a un mayor rendimiento académico que, por otro, la aparición del síndrome de burnout, representado en un mayor agotamiento físico/emocional y cinismo unido a una baja percepción de autoeficacia que suele desembocar en un bajo rendimiento escolar. De esta manera, alude al rol mediador de la autoeficacia en cuanto unos altos niveles de ésta tienden a prevenir las experiencias de estrés y agotamiento conduciendo a un incremento de la percepción de la calidad de vida y satisfacción de los estudiantes frente a la propia institución escolar.

Blanco (2010) sostiene que la autoeficacia académica por parte de los escolares predice su persistencia, dedicación e interés en el estudio al igual que la expectativa de su éxito escolar. Por último, Salanova et al., (2014) mantienen la estrecha relación de las percepciones de autoeficacia y creencias de autorrealización personal con el engagement académico en el desempeño de la actividad escolar de los estudiantes.

Otros trabajos resaltan la estrecha relación entre una baja percepción de autoeficacia de los estudiantes y unos altos niveles de burnout en patrones conductuales más desadaptativos (Durán, Extremadura, Rey, Fernández-Berrocal y Montalbán, 2006; Salanova, Bresó y Schaufeli, 2005). Al mismo tiempo, los estudios de Caballero (2010) y Juarez, Pollora y Sidenius (2006) se centran en las competencias personales necesarias para el correcto desenvolvimiento de los objetivos académicos vinculando aquellos alumnos dedicados, comprometidos y motivados hacia sus estudios con un aumento en sus creencias de autoeficacia y utilización de estrategias metacognitivas a medida que el estrés y agotamiento desaparecen.

Por todo esto, tal y como venimos comentando, aspectos referidos a burnout, engagement y autoeficacia académica influyen y desempeñan una labor relevante sobre las conductas de los estudiantes en edad escolar resultando fundamental atender a ellos para un óptimo desarrollo de la vida académica (Bresó, Schaufeli y Salanova, 2011; Salanova et al., 2014; Vera et al., 2014). 


\section{Limitaciones del estudio}

Las limitaciones del estudio pueden residir en el diseño transversal del mismo tomando los datos en un momento espacio temporal dado. A su vez, los centros de educación secundaria encuestados responden de una manera aleatoria a su inclusión en el estudio no tomando una muestra uniforme de todos los barrios o zonas de la ciudad pudiendo afectar, del mismo modo, a las posibles diferencias entre sus niveles socioeconómicos o educativos así como a otras cuestiones sociales. A la par, los índices de burnout, engagement y autoeficacia académica pueden variar de un curso escolar a otro, e incluso dentro de un mismo año, en cuanto a un cúmulo de circunstancias personales y contextuales del estudiante en su grupo/clase.

\section{Prospectivas de futuro}

Como prospectivas de futuro resultarían interesantes modelos longitudinales que permitiesen valorar la evolución de los constructos estudiados a lo largo de un período más largo de tiempo. Además, sería importante atender otras etapas educativas como pueden ser Educación Primaria o Bachillerato, de la misma manera que con otras etapas de la vida académica del estudiante como pueden ser los Grados de Formación Medio o Superior, así como la propia vida universitaria. Del mismo modo, sería relevante considerar a otras variables relacionadas con las estudiadas como pueden ser el género, el curso académico, la tipología de colegio o el propio rendimiento escolar de los alumnos así como otros aspectos sociales y culturales.

\section{Implicaciones prácticas}

Este trabajo puede tener unas implicaciones de carácter práctico que desemboquen en unas estrategias didácticas a utilizar sobre los alumnos a través de los propios docentes u orientadores escolares desde edades tempranas orientadas hacia conductas predominantemente autodeterminadas como la promoción del esfuerzo, el interés y la motivación en el estudio que hagan a los alumnos sentirse eficaces en el desempeño de sus tareas académicas en aras de una mayor persistencia y dedicación que, a la vez, puedan servir como factor de prevención del bajo rendimiento académico o abandono escolar.

A su vez, programas de intervención dirigidos por profesionales del ámbito educativo en la apuesta de la Administración y/o los propios centros educativos por el trabajo con sus adolescentes en las variables acaecidas, puede ayudar a contribuir a la formación integral y educacional del alumno disminuyendo el prematuro abandono escolar.

Por último, los resultados de nuestra investigación animan a seguir investigando y buscar preguntas que nos ayuden a definir nuevas metodologías encontrando respuestas que nos permitan avanzar en la construcción del desarrollo socioafectivo de los adolescentes escolares.

\section{Referencias}

Asociación Médica Mundial (AMM) (2000). Declaración de Helsinki. Principios éticos para las investigaciones con los seres humanos. Seúl (Corea).

Ato, M., López, J. y Benavente, A. (2013). Un sistema de clasificación de los diseños de investigación en psicología. Anales de psicología, 29(3), 1038-1059. 
Bandura, A. (1999). Ejercicio de la eficacia personal y colectiva en sociedades cambiantes. En: A. Bandura (coord.). Autoeficacia: cómo afrontamos los cambios de la sociedad actual. Bilbao: Desclée de Brouwe.

Blanco, A. (2010). Creencias de autoeficacia de estudiantes universitarios: un estudio empírico sobre la especificidad del constructo. Revista electrónica de investigación y evaluación educativa, 16(1), 1-28.

Brannon, L. (2001). Psicología de la salud. Madrid: Thomson Paraninfo.

Bresó, E. (2008). Well-being and Performance in Academic Settings: The Predicting Role of Selfefficacy (Disertación doctoral no publicada). Universidad Jaume I de Castellón, España.

Bresó, E., Schaufeli, W. B., y Salanova, M. (2011). Can a self-efficacy-based intervention decrease burnout, increase engagement, and enhance performance? A quasiexperimental study. Higher Education, 61, 339-355.

Caballero, C., Hederich, C. y Palacio, J. (2010). El burnout académico. Delimitación del síndrome y factores asociados con su aparición. Revista Latinoamericana de Psicología, 42(1), 131-146.

De Andrade, T.M. (2007). Drug use among young adolescents - prospects for prevention within the context of family relationships and education in the light of damage control principles and practices. Ciencia e Saude Coletiva, 12(5), 1118-1120.

Durán, A., Extremera, N., Rey, L., Fernández-Berrocal, P., y Montalbán, M. (2006). Predicting academic burnout and engagement in educational settings: Assessing the incremental validity of perceived emotional intelligence beyond perceived stress and general self-efficacy. Psicothema, 18, 158-164.

Extremera, N., Durán, A., y Rey, L. (2007). Inteligencia emocional y su relación con los niveles de burnout, engagement y estrés en estudiantes universitarios. Revista de Educación, 342, 239-256.

García, J., Inglés. C., Torregrosa, M., Ruiz, C., Díaz, Á., Pérez, E., Martínez, M. (2010). Propiedades psicométricas de la Escala Autoeficacia Percibida Específica de Situaciones Académicas (EAPESA) en una muestra de estudiantes españoles de Educación Secundaria Obligatoria. European Journal of Education and Psychology, 3(1), 61-73.

Gómez-Fraguela, J.A., Fernández, N., Romero, E. y Luengo, A. (2008). El botellón y el consumo de alcohol y otras drogas en la juventud. Trastornos Adictivos, 20 (2), 211217.

González-Romá, V., Schaufeli, W. B., Bakker, A. B., y Lloret, S. (2006). Burnout and work engagement: Independent factors or opposite poles? Journal of Vocational Behavior, $68,165-174$.

Juarez, A., Pollora, G. y Sidenius, S. (2006). Incidencia del aspecto emocional y socioeconómico: su relación con la diserción. Disponible en: http://www.unl.edu.ar/iberoextension/dvd/archivos/ponencias/mesa1/incidencia-delaspecto-emocional.pdf. Recuperado el 14/X/2017.

Moyano, N. y Riaño-Hernández, D. (2013). Burnout escolar en adolescentes españoles: Adaptación y validación del School Burnout Inventory. Ansiedad y Estrés, 19, 95-103. 
Musitu, G. Jiménez, T. y Murgui, S. (2012). Funcionamiento familiar, autoestima y consumo de sustancias en adolescentes: un modelo de mediación. Revista de salud pública de México, 49(1), 3-10.

Palacio, S., Caballero, C., González, O., Gravini, M. y Contreras, K. (2012). Relación del burnout y las estrategias de afrontamiento con el rendimiento académico en estudiantes universitarios. Universytas Psychological, 11(2), 178-186.

Parra, P. (2010). Relación entre el nivel de engagement y el rendimiento académico teórico y práctico. Revista Educación Ciencias de la Salud, 7(1), 57-63.

Rodriguez-García, A.M., Sola-Martínez, T. y Fernandez-Cruz, M. (2017). Impacto del burnout en el desarrollo profesional del profesorado universitario. Una revisión de la investigación. Revista universitaria de Formación del Profesorado, 20(3). 161-178. DOI: http://dx.doi.org/10.6018/reifop.20.3.275121

Salanova, M., Bresó, E., y Schaufeli, W. B. (2005). Hacia un modelo espiral de las creencias de eficacia en el estudio del burnout y del engagement. Ansiedad y Estrés, 11, 215-231.

Salanova, M., Del Líbano, M., Llorens, S., y Schaufeli, W. B. (2014). Engaged, workaholic, burned-out or just 9-to-5? Toward a typology of employee well-being. Stress and Health, 30, 71-81.

Salanova, M., Martínez, I. M., Bresó, E., Llorens, S., y Grau, R. (2005). Bienestar psicológico en estudiantes universitarios: facilitadores y obstaculizadores del desempeño académico. Anales de Psicología, 21, 170-180.

Salanova, M., Martínez, I. M., y Llorens, S. (2014). Una mirada más" positiva" a la salud ocupacional desde la Psicología Organizacional Positiva en tiempos de crisis: aportaciones desde el equipo de investigación WoNT. Papeles del psicólogo, 35, 2230.

Salanova, M., Schaufeli, W., Martínez, I. y Bresó, E. (2009). How obstacles and facilitators predict academic performance: The mediating role of study burnout and engagement. Anxiety, Stress and Coping, 23, 53-70.

Salavera, C. y Usán, P. (2017). Repercusión de las estrategias de afrontamiento de estrés en la felicidad de los alumnos de Secundaria. Revista electrónica interuniversitaria de formación del profesorado, 20(3), 65-77. DOI: http://dx.doi.org/10.6018/reifop.20.3.282601

Schaufeli, W. y Bakker, A., (2004) Job demands, job resources, and relationship with burnout and engagement: a multisample study. Journal of Organizational Behavior. Vol. 25 Issue 3, 293.

Schaufeli, W., Martínez, I., Marques-Pinto, A., Salanova, M. \& Bakker, A. (2002). Burnout and engagement in university students: A crossnational study. Journal of Cross-Cultural Psychology, 33(5), 464-481.

Schaufeli, W., Salanova, M., González-Romá, V., y Bakker, A. (2002). The measurement of burnout and engagement: A confirmatory factor analytic approach. Journal of Happiness Studies, 3(1), 71-92.

Schauffeli, W. y Salanova, M. (2007). Efficacy or inefficacy, that's the question: Burnout and work engagement, and their relationships with efficacy believes. Anxiety, Stress and Coping, 20, 177-196. 
Seligman, M. y Csikszentmihalyi, M. (2000). Positive psychology: An introduction. American Psychologist, 55, 5-14.

Seligman, M., Steen, T., Park, N. y Peterson, C. (2005). Positive psychology progress. Empirical validation of interventions. American Psychologist, 60, 410- 421.

Vera, M., Le Blanc, P., Taris, T. y Salanova, M. (2014). Patterns of engagement: the relationship between efficacy beliefs and task engagement at the individual versus collective level. Journal of Applied Social Psychology, 44, 133-144. 